\title{
ElderReact: A Multimodal Dataset for Recognizing Emotional Response in Aging Adults
}

\author{
Kaixin $\mathrm{Ma}^{*}$ \\ Carnegie Mellon University \\ Pittsburgh, Pennsylvania \\ kaixinm@andrew.cmu.edu \\ Mingtong Zhang \\ Carnegie Mellon University \\ Pittsburgh, Pennsylvania \\ mingtonz@andrew.cmu.edu
}

\author{
Xinyu Wang* \\ Carnegie Mellon University \\ Pittsburgh, Pennsylvania \\ xinyuw3@andrew.cmu.edu \\ Jeffrey M. Girard \\ Carnegie Mellon University \\ Pittsburgh, Pennsylvania \\ jmgirard@cmu.edu
}

\author{
Xinru Yang \\ Carnegie Mellon University \\ Pittsburgh, Pennsylvania \\ xyang@andrew.cmu.edu
Louis-Philippe Morency
Carnegie Mellon University
Pittsburgh, Pennsylvania
morency@cs.cmu.edu

\begin{abstract}
Automatic emotion recognition plays a critical role in technologies such as intelligent agents and social robots and is increasingly being deployed in applied settings such as education and healthcare. Most research to date has focused on recognizing the emotional expressions of young and middleaged adults and, to a lesser extent, children and adolescents. Very few studies have examined automatic emotion recognition in older adults (i.e., elders), which represent a large and growing population worldwide. Given that aging causes many changes in facial shape and appearance and has been found to alter patterns of nonverbal behavior, there is strong reason to believe that automatic emotion recognition systems may need to be developed specifically (or augmented) for the elder population. To promote and support this type of research, we introduce a newly collected multimodal dataset of elders reacting to emotion elicitation stimuli. Specifically, it contains 1323 video clips of 46 unique individuals with human annotations of six discrete emotions: anger, disgust, fear, happiness, sadness, and surprise as well as valence. We present a detailed analysis of the most indicative features for each emotion. We also establish several baselines using unimodal and multimodal features on this dataset. Finally,
\end{abstract}

\footnotetext{
${ }^{*}$ Both authors contributed equally to this research.
}

Permission to make digital or hard copies of all or part of this work for personal or classroom use is granted without fee provided that copies are not made or distributed for profit or commercial advantage and that copies bear this notice and the full citation on the first page. Copyrights for components of this work owned by others than the author(s) must be honored. Abstracting with credit is permitted. To copy otherwise, or republish, to post on servers or to redistribute to lists, requires prior specific permission and/or a fee. Request permissions from permissions@acm.org. ICMI '19, October 14-18, 2019, Suzhou, China

(c) 2019 Copyright held by the owner/author(s). Publication rights licensed to ACM.

ACM ISBN 978-1-4503-6860-5/19/10 ..\$15.00

https://doi.org/10.1145/3340555.3353747 we show that models trained on dataset of another age group do not generalize well on elders.

\section{CCS CONCEPTS}

- Human-centered computing $\rightarrow$ Human computer interaction (HCI); • Applied computing $\rightarrow$ Psychology.

\section{KEYWORDS}

Emotion Recognition, elders, nonverbal behavior analysis

\section{ACM Reference Format:}

Kaixin Ma, Xinyu Wang, Xinru Yang, Mingtong Zhang, Jeffrey M. Girard, and Louis-Philippe Morency. 2019. ElderReact: A Multimodal Dataset for Recognizing Emotional Response in Aging Adults . In 2019 International Conference on Multimodal Interaction (ICMI '19), October 14-18, 2019, Suzhou, China. ACM, New York, NY, USA, 9 pages. https://doi.org/10.1145/3340555.3353747

\section{INTRODUCTION}

Automatic emotion recognition plays a central role in various technologies such as conversational agents [28] and social robots [17]. Successful emotion recognition serves as key foundation for adaptive systems which make more appropriate response based on users' emotions [37][29]. Moreover, automatic emotion recognition has gained popularity in education applications such as affect-aware tutors [23] which personalize teaching strategy based on learner's emotion states as well as health care technologies which facilitate doctors' assessment of potential psychological disorder [36][39].

Despite numerous efforts on automatic emotion recognition, most of the work has focus on adults, and to a lesser extent, children. Very few studies have focused on elders, which represent a large and growing population worldwide [1][25]. With the rise of elder population, the need for technologies such as socially assistive robots also surges. Such technologies build upon accurate automatic emotion recognition system, thus we ought to pay more attention on emotion recognition for elders specifically. However, this task can be 


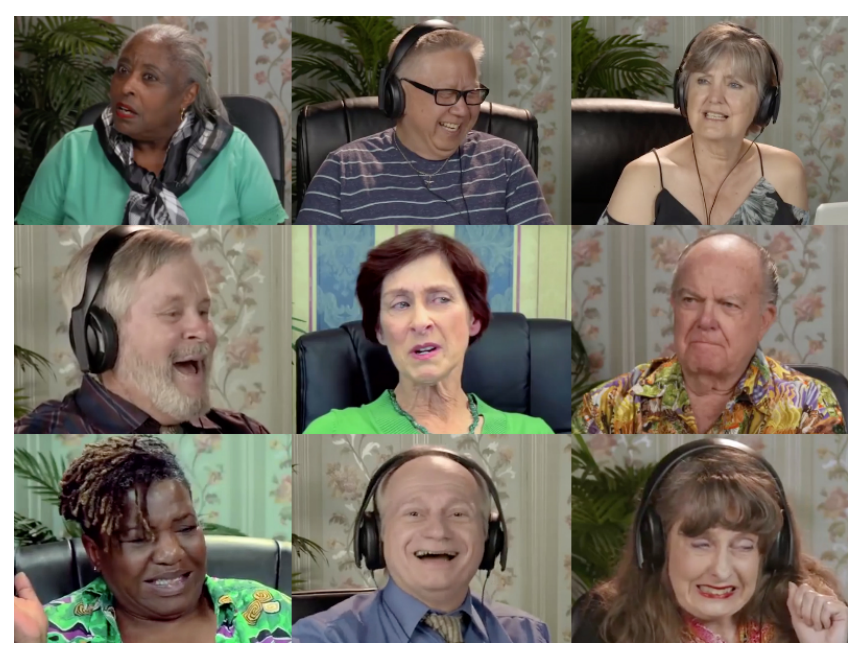

Figure 1: Example frames on ElderReact dataset, showing various facial expressions

very challenging because aging changes facial shape and has been known to alter nonverbal behavior as well [24] [14]. Moreover, there are very few publicly available datasets; only one contains videos and another one contains speech [42] [43], which prohibits effective training of computational models.

To address aforementioned challenges, we introduce a newly collected multimodal dataset for building automatic recognition systems for elders - ElderReact. The dataset contains 1323 video clips of 46 unique individuals and each of the video clips is annotated 6 emotion states: anger, disgust,fear, happiness, sadness, and surprise as well as valence. Figure 1 shows example frames from our dataset. We conduct statistical analysis to identify the most indicative features for each emotion and provide insights for predictive modeling. We then build several baselines predictive models on our dataset using unimodal and multimodal features to recognize these affects automatically. Finally, to investigate the impact of age difference on computational models for emotion recognition, we train our models on ElderReact and EmoReact[3], a dataset designed for studying children's emotions and performed cross testings. We used these dataset to explore how well a model trained on a certain age group can generalize to another one.

\section{RELATED WORK}

Despite the importance of emotion recognition in elder people, there are very few datasets built specifically for that purpose. Most datasets including aging adults are used for studying age progression and age estimation, or for building more robust models that can recognize faces in an age invariant way [16].
For age progression task, the most widely used benchmark datasets are the Face and Gesture recognition Network database (FG-NET) [27] and the MORPH dataset (album 2) [31]. The FG-NET database contains 1,002 facial images from 82 different subjects with ages ranging from newborns to 69 years old. However, most of the face images are of ages from 0 to 40 years [22]. The MORPH dataset (album 2) contains 55,134 facial images of 13,618 people. Nevertheless, only 3,984 images are of ages more than 50 years old [30]. Other popular datasets with age annotation include OUI-adience [10], IMDB-wiki [33], where elder people are still less represented: in OUI-adience dataset, only 869 out of $26 \mathrm{k}$ images are of people more than 60 years old.. The newly collected AgeDB [26] dataset contains more images of elder faces. It has 16,488 facial images of 568 subjects and the average age range for each person is 50.3 years. Roughly, 40 percent of the images are of faces more than 50 years old. While it contains a considerable amount of facial images of the elders, no multimodal data is available. Previous study [5] shows that multimodal data is helpful for emotion recognition and the lack of them makes AgeDB less suitable for emotion recognition task.

In common emotion recognition datasets, elder people are even less represented. Popular multimodal emotion recognition datasets include SEWA database [20], REmote COLlaborative and Affective interactions (RECOLA) database [32], AVEC 13 dataset [40] and Acted Facial Expressions in the Wild (AFEW) dataset [9]. The SEWA database contains 398 unique subjects, with only 30 individuals being more than 60 years old. In AVEC 13 dataset, there are 292 subjects but less than 5\% of the individuals are from 56-63 years old by approximate (only mean age, standard deviation and age range are provided). The AFEW dataset has similar annotated labels to our dataset (anger, disgust, fear, happiness, sadness, surprise, or neutral) and 330 subjects, but only provides age range information without specifying the portions of each age group. In RECOLA dataset, no age information is provided.

The only datasets we know dedicating to elder emotion recognition are the Elderly Emotional Speech Database (EESDB) [42] and the Emotional Interaction Database of Speech and Video signal Database (EISVDB) [43]. The EESDB dataset contains 800 speech utterances covering seven classes of emotions (anger, disgust, fear, happiness, neutrality, sadness and surprise). There are eight male and eight female individuals, all of them are actors and are over 60 years old. The EISVDB dataset contains 810 utterances from sixteen individuals and includes both audio data and video clips. It covers seven kinds of emotions (anger, boredom, happiness, sadness, surprise, neutrality and disgust). However, the EESDB 
Table 1: Annotation Reliability Measurements, where $A=$ Agreement, $S=$ Bennett et al. $S, \alpha=$ Krippendorff $\alpha$, ICC = Intraclass Correlation and Finn $=$ Finn Reliability

\begin{tabular}{lccccccc}
\hline & \multicolumn{3}{c}{ Binary } & & \multicolumn{2}{c}{ Ordinal } \\
\cline { 2 - 3 } \cline { 7 - 8 } Emotion & $A$ & $S$ & $\alpha$ & & ICC & FINN \\
\hline Anger & 0.72 & 0.44 & 0.34 & & 0.64 & 0.76 \\
Disgust & 0.79 & 0.57 & 0.43 & & 0.78 & 0.79 \\
Fear & 0.83 & 0.65 & 0.33 & & 0.68 & 0.87 \\
Happiness & 0.79 & 0.58 & 0.58 & & 0.85 & 0.69 \\
Sadness & 0.73 & 0.47 & 0.26 & & 0.60 & 0.77 \\
Surprise & 0.64 & 0.29 & 0.28 & & 0.66 & 0.64 \\
Valence & - & - & - & & 0.83 & 0.76 \\
\hline
\end{tabular}

dataset only focus on audio modalities and both dataset contain only 16 individuals, which makes it hard to build person independent models.

To the best of our knowledge, the ElderReact dataset is the largest multimodal dataset dedicated for studying elders emotions. It contains a large group of elders and provides multimodal information that can be useful for automatic emotion recognition. In addition, it can be good resources for studies on elder emotion expression analysis and emotion intensity analysis.

\section{DATASET}

In this section, we first describe the process of raw data collection. Then we outline the emotion annotation procedure. Finally we present dataset statistics and some basic analysis. ${ }^{1}$

\section{Data collection}

We first collected 43 videos from the YouTube REACT channel in which elders react to different stimulus. These videos' stimulus cover a wide range of topics including video games, social events and online challenges. These videos typically have 3 stages: (1) The elders are first told the stimuli they will be reacting to and asked what they know about the stimuli, (2) elders are presented the stimuli and they interact with the stimuli, (3) elders are asked their opinions about the stimuli after getting to know more.

The original videos contain multiple people reacting to the stimuli. We used the open source tool PySceneDetect ${ }^{2}$ to automatically segment videos into short clips so that each clip only have one person's reaction. We only kept clips that are at least 3 seconds and manually filtered out clips with low quality, i.e. some scene change may be omitted by

\footnotetext{
${ }^{1}$ All of the videos, annotations and processed features are available to public for research use and can be accessed at https:/github.com/Mayer123/ ElderReact

${ }^{2}$ https://github.com/Breakthrough/PySceneDetect
}

Table 2: Occurrence of each emotion in videos and people

\begin{tabular}{lcc}
\hline Emotion type & Videos & People \\
\hline Anger & $350(26 \%)$ & $39(85 \%)$ \\
Disgust & $278(21 \%)$ & $36(78 \%)$ \\
Fear & $152(11 \%)$ & $28(61 \%)$ \\
Happiness & $742(56 \%)$ & $44(96 \%)$ \\
Sadness & $241(18 \%)$ & $38(83 \%)$ \\
Surprise & $566(43 \%)$ & $45(98 \%)$ \\
\hline
\end{tabular}

PySceneDetect so that more than one person would end up in one clip. This process generated 1,323 clips in total and these clips are typically 3 to 8 seconds long $(M=5.9 \mathrm{~s}, \mathrm{SD}=2.9 \mathrm{~s})$. Additionally, we manually identified videos from the same individual, i.e grouped videos by person. In total we have 46 people in our dataset in which 26 are female and 20 are male.

\section{Emotion Annotations}

From our pilot study, we noticed that some of the complex emotions such as curiosity rarely appear in our videos. Thus we decided to only focus on 6 basic emotions [11]: Anger, Disgust, Fear, Happiness, Sadness and Surprise. We also annotated the valence that is helpful for analysis of emotion desirability.

We used the crowd-source platform - Amazon Mechanical Turk to collect annotations. Each clip was annotated by 3 workers. For each emotion label, we asked a compound question about the existence of that emotion (We derived these questions from the Discrete Emotions Questionnaire[18]). For example, for emotion Anger, we would ask "How an$\mathrm{gry} / \mathrm{mad} /$ frustrated was the individual in the video?". Workers would answer questions about emotions on a 1-4 scale where 1, 2, 3, 4 represent "Not at all", "Slightly", "Moderately" and "Very much" respectively. We asked workers to judge valence of the video on a 1-7 scale where 1 means very negative and 7 means very positive. Since we observed that multiple emotions may appear in one video clip, i.e mixed emotions or transitioning emotions, we asked workers to rate all emotions for each video clip. Additionally, we asked the worker to annotate the gender of the individual in the video to see if the worker was actually paying attention. We required all workers to be in an U.S. location, have lifetime approval rate greater than $97 \%$ and have more than 500 HITs (Human Intelligence Task) approved before. All HITs in which gender of the individual in the video was answered incorrectly were rejected and new workers would be asked to provide substitute annotations. 


\section{Annotation Statistics}

To evaluate the quality of those annotations, we first compute intra-class correlation scores [35] and Finn's metric[13] on raw ratings to estimate how consistent the annotations are among videos. The scores are shown in the right half of the table 1 . Considering a score no less than 0.75 to be good and a score no less than 0.6 to be acceptable, workers' rating are good for disgust, happiness, and valence and acceptable for all the others with ICC estimates. Using the Finn estimates, workers' rating are good for anger, disgust, fear, sadness and valence, and acceptable for happiness and surprise.

Then we convert the raw ratings of workers into binary values by mapping 1 as not present, 2 and larger values as present. We then compute observed agreement, SScore[4] and Kripendorff alpha[21] on binary scores to evaluate the agreement among workers. The agreement scores are shown in the left half of the Table 1 . As we can see that the observed agreement for all emotions and valence are relatively high. However, when taking into account random chance agreement, the agreement scores become much lower. The Kripendorff alpha is lower than SScore mainly due to the data imbalance issue. For example, there are only $18 \%$ of videos containing sadness, hence the alpha would assign high probability to chance agreement, resulting low final score.

Then we finalize the labels for each video by taking the majority voting of all annotations. The number of videos for each emotion and the number of people who have shown the emotion are presented in Table 2. Although there is still imbalance among emotions, we get a good number of samples for each of the emotion and each emotion is expressed by many people, which should allow researcher to build more computational prediction models and study the generalization ability to unseen individuals.

Since each clip may contains many emotions, we also plot the co-occurrence patterns of emotions in Figure 2. We normalize each cell by the number of videos of emotions on the $\mathrm{x}$-axis. It is worth noting that Figure 2 is not symmetrical because corresponding cells are normalized against different emotions occurrences. For example, assume that the co-occurrence between anger and disgust is $\mathrm{x}$, occurrences of anger is $y$ and occurrences of disgust is $z$. Then on anger and disgust column, the numbers will be $\mathrm{x} / \mathrm{y}$ and $\mathrm{x} / \mathrm{z}$ respectively. These numbers give us a sense of how frequent a particular co-occurrence type happens when a certain emotion exists. It's interesting to note that happiness co-occurred with surprise very often. We think the main reason is that in many of our videos, the elders are asked to react to things that they're unfamiliar with such as video games but they also enjoy them a lot. On the other hand, surprise also co-occurred with fear

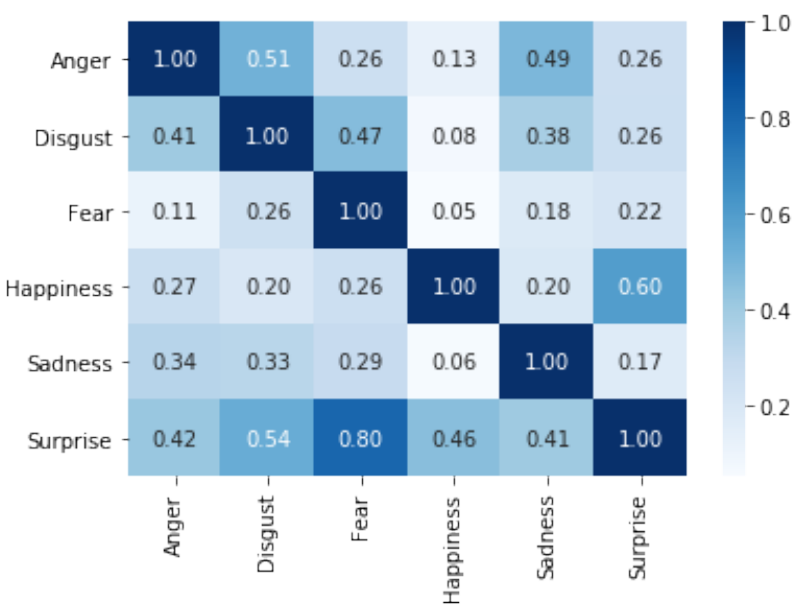

Figure 2: Co-occurrence between different Emotion states in elder people

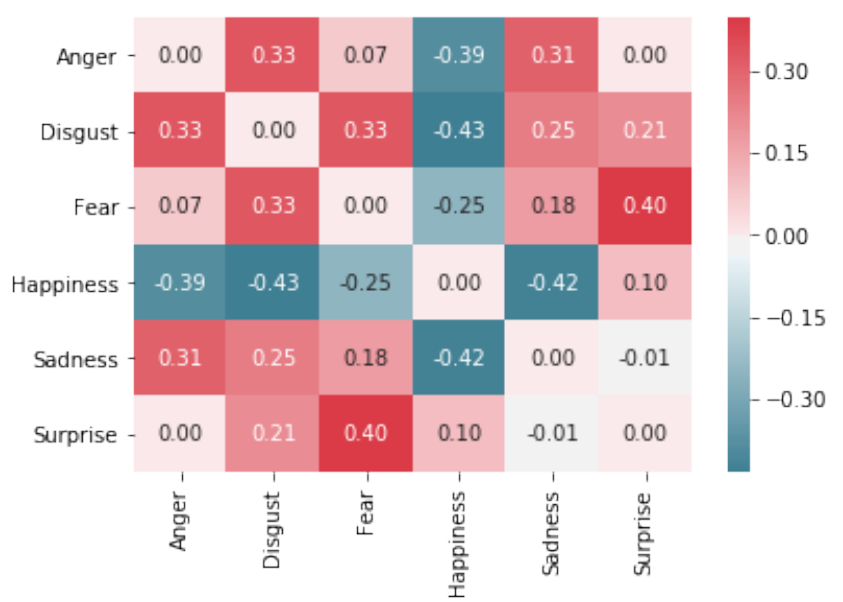

Figure 3: Correlation of different emotion states in elder people

quite often. When elders are asked to react to online challenges, e.g watching a video of hundreds of spiders crawling on the wall, the contents are often unexpected for them and they are afraid of what they saw, resulting the relative high co-occurrence rate of fear with surprise.

We're also interested in the correlation between emotions, thus we averaged 3 annotations' ratings for emotions in each video and computed Pearson correlation between emotions. The results are shown in Figure 3, which provides us another view of the dataset. Similar to co-occurrence pattern, high correlation exists between surprise and fear. Negative correlation are observed between happiness and anger, disgust, fear and sadness respectively, which may not be obvious in co-occurrence pattern. Moreover, moderate correlation are 
observed between negative emotion pairs such as disgust with anger. The reason is that many of our videos' stimulus are social phenomena such as bullying, which are likely to elicit these emotions together.

\section{STATISTICAL ANALYSIS}

In this section, we describe the multimodal behavioral features we extracted from our dataset and provide our analysis about the most indicative visual and audio feature of each of the emotion states. We followed previous work to design our feature template [3].

For visual features, we extract the following 4 types:

(1) Gaze: These features describe the directions of eye gaze on 3D axis as well as the angle shift (left to right or down to up). We observed in our data that people tend to look away when they are disgusted, suggesting the potential usefulness of gaze features. Therefore, we extracted 8 gaze-related features.

(2) Head Pose The features of head pose provide information about the location of head with respect to the camera in 3D space as well as head movement (head nods or head shakes). We think that head shakes are salient indicators for emotions such as disgust based on our observation. 6 pose-related features are extracted.

(3) Facial Action Unites(FAU): These features describe the most basic independent facial muscle movements, which are known to be strong indicators of emotions [12]. In total we extracted 35 FAU features.

(4) Non-rigid Shape Parameters: These feature come from applying PCA on facial landmarks and they describe facial deformation due to expression and identity (widening the eyes or opening mouth), which can be useful for emotion such as fear [3]. We have 50 features for this type.

For audio features, we extract the following 3 types:

(1) Voice quality: The voice quality features such as normalized amplitude quatient (NAQ) are extracted which describe speech characteristics such as tenseness, creakiness and breathiness. 11 voice quality features are extracted.

(2) MFCC: The feature of Mel-Frequency Cepstral Coefficient is widely accepted in speech processing community and has been successfully applied in emotion recognition task [34]. We extracted MFCC 1-24 for our analysis and experiments.

(3) Prosody: The fundamental frequency or the pitch of the person's voice.

\section{Feature Analysis}

We performed t-test to identify the most indicative visual and audio features for each of the emotions. In our annotation
Table 3: Most indicative visual features for each emotion, where $M=$ mean and $S D=$ standard deviation

\begin{tabular}{llcl}
\hline Emotion & Feature & Stat & Hedge's g \\
\hline \multirow{2}{*}{ Anger } & Lip corner depressor & SD & 0.98 \\
& Lip stretcher & SD & 0.97 \\
Disgust & H Pose Shift & SD & 1.01 \\
& H Gaze Shift & SD & 0.91 \\
Fear & Blink & SD & -2.00 \\
& Chin raiser & SD & -2.13 \\
Happiness & Cheek raiser & SD & 1.23 \\
& Lip corner puller & SD & 1.50 \\
Sadness & P 16 & SD & 0.89 \\
Surprise & V Gaze & M & 0.54 \\
& V Gaze angle & M & 0.56 \\
\hline
\end{tabular}

Table 4: Most indicative audio features for each emotion, where $M=$ mean and $S D=$ standard deviation

\begin{tabular}{llcl}
\hline Emotion & Feature & Stat & Hedge's g \\
\hline Disgust & MFCC 0 & M & 1.20 \\
& MFCC 2 & M & -1.03 \\
Fear & F0 & M & 1.50 \\
Happiness & F0 & M & 1.00 \\
& MFCC 2 & M & -1.09 \\
Sadness & NAQ & SD & 0.61 \\
Surprise & MFCC 0 & M & 0.88 \\
\hline
\end{tabular}

task, we asked a compound question for every emotion to maximize the coverage. In some of our videos, none of the emotions are identified. Then we assume that these videos represent neutral expressions, therefore we use these videos as the control group. We compare this group of videos against the group of videos that only contain the emotion we test. We also average all instances of the same individual for both group to reduce any potential bias. Additionally, we report the Hedge's $g$ to indicate the effect size of the significant features [19]. We used p-value of 0.05 to be our threshold ${ }^{3}$. The most indicative visual features are shown in Table 3 and the most indicative audio features are shown in Table 4.

We get some intuitive results from the feature analysis. For example, the horizontal gaze and pose shift are identified as important feature for disgust, which correspond to our observation that people often look away when they see disgusting stimuli. Also blink and chin raiser were found to have very large negative effect on fear, which means that when people are fear they would blink a lot less and have a

\footnotetext{
${ }^{3}$ Since we only meant to get a sense of which features are important, we did not perform multiple comparisons correction
} 
lower chin. For audio features, we did not find any significant features for anger, we think the reason might be that individuals in our dataset are not raging but are upset, so they would still speak in a calm tone. We also observe that in many of the fear cases individuals would scream, which also correspond that F0 was found to be an important indicator of fear.

\section{EXPERIMENTAL SETUP}

\section{Data split}

We separated our dataset into three subsets: training set, which contains 615 videos; validation set, which has 355 videos; and test set, which has 353 videos. These sets are defined in a person independent manner. The number of videos associated with each individual varies quite a lot (Min=2, Max=154). Previous study also suggests that unique characteristics of certain individuals can be a confounding factor for model training on sentiment classification task [41]. For our case, the model may learn to classify an emotion based on a person's characteristic if that person has only shown one emotion in all of his/her videos. Thus we put individuals with the most videos (diverse emotion labels) into training set to reduce the variance from appearance and ones with least videos (more individuals) into test set to test the robustness of the models. Specifically, training set has 8 individuals, dev set has 8 individuals and test set has 30 individuals. We hope this split could encourage researchers to develop models that have better generalizability.

\section{Feature Extraction}

We extracted visual features using the open-source tool OpenFace $[2]^{4}$. We selected frames where the faces are successfully detected. Overall, $97.9 \%$ of the frames in our dataset are successfully processed by OpenFace. We used another open-source tool COVAREP $[8]^{5}$ to extract audio features using frame length of 10 milliseconds. After extracting the raw features for each frame, we summarize the video clip for both modalities by computing the mean and standard deviation for all frames.

\section{Baseline Models}

We built several baselines including Gaussian Naive Bayes [15], Radial Basis Function kernel Support Vector Machine [38] and XGBoost [6] as our baseline models. We also include random baseline. We report raw F1 scores for all of our models. Additionally, we calculated Cohen Kappa scores [7] of XGBoost's results and compared them to human agreement scores to see the upper-bound of the model performance.

\footnotetext{
${ }^{4}$ https://github.com/TadasBaltrusaitis/OpenFace

${ }^{5}$ https://github.com/covarep/covarep
}

\section{Implementation Details}

We performed min-max feature normalization since the ranges of values for raw features differ. We used the validation set for tuning the hyper-parameters of SVM. To handle the problem of imbalanced emotion labels, we performed under-sampling and used ensemble classifiers following previous work [3]. Specifically, we first randomly selected a subset of negative examples that has the same size as positive examples and used the subset along with positive examples as training data. We then trained a classifier and got its prediction. Then we repeated the process for 100 times and used the majority voting method to decide the final predictions. The same approach is used for all the baseline models (i.e. all the models are ensembles of 100 classifiers except for the random baseline). We used only one group of features (audio or visual) for unimodal experiments and early fusion (concatenated the audio and visual features and used the new feature sets as input) for multimodal experiments.

\section{Cross-dataset generalization}

One of the most important reasons that we do need an emotion dataset for elder is that, the systems built on other age groups may not perform as well on elders. To investigate the impact of age difference on computational models for emotion recognition, we conducted cross-dataset experiments. Specifically, we used EmoReact [3], a multimodal emotion dataset designed for emotion recognition task for children. Although children exhibit a larger difference from elders than middle-aged adults in appearance, the EmoReact dataset has the most similar settings to our dataset (e.g. similar background in the scene, lighting, video clips of similar length) which allow us to isolate the variable we are interested in: age. Thus EmoReact can be a good comparison technicalwise.

We trained SVM classifiers (using the approaches mentioned before) on both EmoReact and ElderReact dataset and tested on the corresponding test sets. Then, we swapped the training sets and conducted the evaluations again, using the same hyper parameters as with original training set. We used both audio and visual features as input and evaluate the model performance using F1 score. Note that there are only four overlapping emotions among these datasets so F1 scores on only four affect states are reported.

\section{RESULTS AND DISCUSSION}

\section{Baseline results}

Table 5 shows results from our baseline models. All models outperform random baseline on all emotion states and the best model outperform by a large margin. XGBoost stands out as the best model for all emotions except fear. SVM's performance is very close to that of XGBoost on all emotions. 
Table 5: F1 scores for each emotion in ElderReact, where Ang means Anger, Dis means Disgust, Fea means Fear, Hap means Happiness, Sad means Sadness and Sur means Surprise

\begin{tabular}{lcccccc}
\hline Model & Ang & Dis & Fea & Hap & Sad & Sur \\
\hline Random & 0.30 & 0.26 & 0.14 & 0.51 & 0.27 & 0.41 \\
Naive Bayes & 0.34 & 0.27 & $\mathbf{0 . 2 5}$ & 0.56 & 0.33 & 0.45 \\
SVM & 0.41 & 0.35 & 0.16 & 0.70 & 0.34 & $\mathbf{0 . 5 4}$ \\
XGBoost & $\mathbf{0 . 4 3}$ & $\mathbf{0 . 3 6}$ & 0.17 & $\mathbf{0 . 7 1}$ & $\mathbf{0 . 3 5}$ & $\mathbf{0 . 5 4}$ \\
\hline
\end{tabular}

Table 6: Comparison of our best model with the human agreement scores on Cohen's Kappa

\begin{tabular}{lcccccc}
\hline Model & Ang & Dis & Fea & Hap & Sad & Sur \\
\hline XGBoost & 0.19 & 0.14 & 0.04 & 0.38 & 0.11 & 0.22 \\
Human & $\mathbf{0 . 2 9}$ & $\mathbf{0 . 3 9}$ & $\mathbf{0 . 2 2}$ & $\mathbf{0 . 5 5}$ & $\mathbf{0 . 2 8}$ & $\mathbf{0 . 2 3}$ \\
\hline
\end{tabular}

Naive Bayes does not perform as good as these classifiers, which is understandable, as Naive Bayes classifier assumes the independence among predictors.

\section{Comparison to human agreement}

We also compared our best model's results to the human agreement scores on Cohen's Kappa metric. As shown in table 6, all emotions except for surprise exhibit much lower scores than human's, suggesting that there is still large room for improvement.

\section{Performance using different feature sets}

Figure 4 shows the results of the SVM classifier using different set of features: audio only, visual only and early fusion.

For anger, disgust, happiness and sadness, the visual-only setting outperform audio-only setting by a large margin whereas audio-only setting results are comparable to visualonly results for fear and surprise. Overall, the visual features are more indicative than audio features according to our experiments. We hypothesize that elder people will moderate themselves when expressing emotions. When doing so, they can control their tones more easily than control facial expressions so that it's easier to use visual features to recognize emotions.

For anger, happiness and surprise, early-fusion setting achieves the best performance. On the other hand, early fusion's results are comparable to that of visual-only setting for disgust, lags from visual-only settings for sadness, and are lower than both visual-only and audio-only settings for fear. The reason may be that for anger, happiness and surprise, the facial expressions are more subtle. For example, a person may only have a gentle smile when being happy, making it

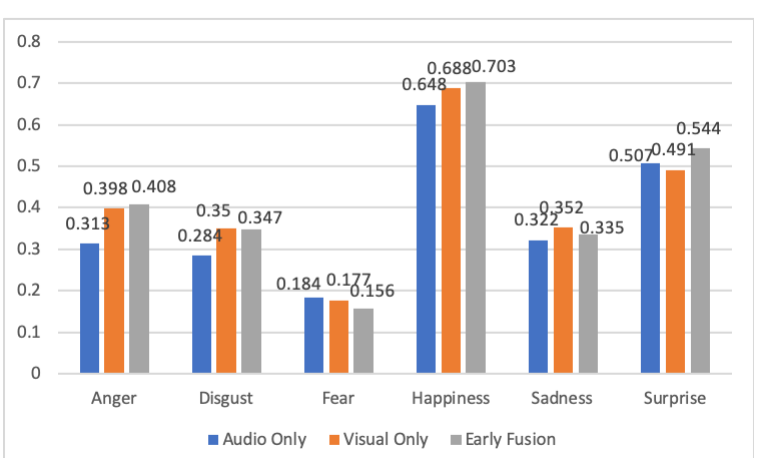

Figure 4: SVM performance (F1 scores) with different feature sets

difficult to recognize happiness. In this case, audio features can be complementary to visual features. For sadness, people can be very clear in expressing these emotions using facial expressions. In contrast, they may not be speaking much when they are feeling sad, making the audio signals more like noises than useful features.

\section{Cross-dataset generalization results}

Figure 5 shows the performance differences when training on either children/elder data and testing on elder dataset. Figure 6 shows the performance differences when training on either children/elder data and testing on children dataset. We can see that the performance drop significantly when training on one dataset and testing on the other for all emotion states.

It is interesting to note the case for happiness. The model performance drops only moderately when training on elder data and testing on children data, but drops significantly when training on children data and testing on elder data. This means that the model trained on elder data is more robust regarding recognizing happiness. It can be due to the fact that children have more facial and head movements when being happy, such that the noises are also learned by the model. On the contrary, the model is more likely to learn the truly indicative features (e.g. raising the cheek) when using elder data for training.

\section{Limitations}

Automatic emotion recognition for elders is an important but challenging task and we provide this dataset to the community to better study this task. However, we recognize that there are certain limitations on our work. First, our data source - Youtube reaction channel, is designed for entertainment purpose. Thus the participants may have exaggerated emotional response, which may not perfectly reflect the real world scenario. So it would be worthwhile to collect more data from daily life settings in the future. Secondly, although our dataset is the largest one designed for studying elders' 


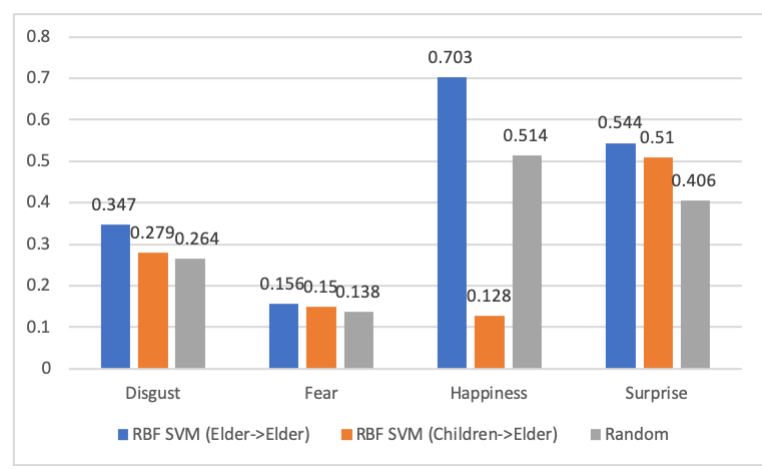

Figure 5: F1 scores with cross testing on ElderReact

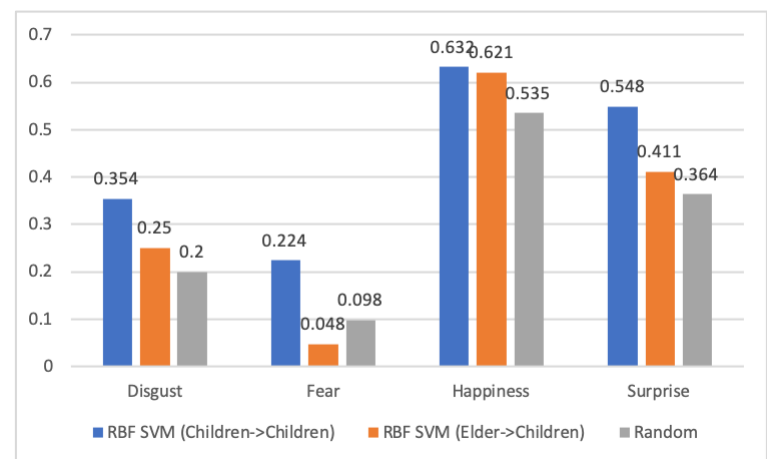

Figure 6: F1 scores with cross testing on EmoReact

emotional response, it is not large enough for effectively training deep neural network models. It would be interesting to collect additional data and apply deep learning approaches on this task. Finally, in our cross-dataset experiments, we utilize the EmoReact dataset which contains children's emotional response. Since children's facial muscles and voices differ from elders more than adults do, it would be good to conduct cross-dataset experiments with an adult dataset. Thus, we would like to collect adult data with similar experimental setup (background, lighting, etc.) in the future.

\section{CONCLUSIONS}

The main contribution of this paper is the introduction of the multimodal dataset, ElderReact for building automatic emotion recognition systems for elders which contains 1323 videos clips annotated with 6 emotions and valence. We also present an analysis of the most indicative visual and audio features for each emotion. Our experiments indicate that although baseline models have shown promising performance, there are still large room for improvement. In addition, based on cross-dataset generalization experiments, we observe that models trained on one age group has trouble generalizing on the other. Thus models need to specifically designed for each age group to get better performance.

\section{ACKNOWLEDGMENTS}

We thank Keith Maki, Alexandria Vail, Zhun Liu for helpful suggestions on this work and the anonymous reviewers for their valuable feedback.

\section{REFERENCES}

[1] Gerard F. Anderson and Peter Sotir Hussey. 2000. Population Aging: A Comparison Among Industrialized Countries. Health Affairs 19, 3 (2000), 191-203. https://doi.org/10.1377/hlthaff.19.3.191 arXiv:https://doi.org/10.1377/hlthaff.19.3.191

[2] Tadas Baltrusaitis, Peter Robinson, and Louis-Philippe Morency. 2016. OpenFace: An open source facial behavior analysis toolkit.. In $W A C V$. IEEE Computer Society, 1-10. http://dblp.uni-trier.de/db/conf/wacv/ wacv2016.html\#Baltrusaitis0M16

[3] Charles E Hughes Behnaz Nojavanasghari, Tadas Baltrusaitis and Louis-Philippe Morency. 2016. EmoReact: a multimodal approach and dataset for recognizing emotional responses in children. Proceedings of the 18th ACM International Conference on Multimodal Interaction (2016).

[4] E. M. BENNETT, R. ALPERT, and A. C. GOLDSTEIN. 1954. Communications Through Limited-Response Questioning*. Public Opinion Quarterly 18, 3 (01 1954), 303-308. https://doi.org/10.1086/266520 arXiv:http://oup.prod.sis.lan/poq/article-pdf/18/3/303/5384778/18-3303.pdf

[5] Carlos Busso, Zhigang Deng, Serdar Yildirim, Murtaza Bulut, Chul Min Lee, Abe Kazemzadeh, Sungbok Lee, Ulrich Neumann, and Shrikanth Narayanan. 2004. Analysis of Emotion Recognition Using Facial Expressions, Speech and Multimodal Information. In Proceedings of the 6th International Conference on Multimodal Interfaces (ICMI '04). ACM, New York, NY, USA, 205-211. https://doi.org/10.1145/1027933.1027968

[6] Tianqi Chen and Carlos Guestrin. 2016. XGBoost: A Scalable Tree Boosting System. In Proceedings of the 22Nd ACM SIGKDD International Conference on Knowledge Discovery and Data Mining (KDD '16). ACM, New York, NY, USA, 785-794. https://doi.org/10.1145/2939672.2939785

[7] Jacob Cohen. 1960. A Coefficient of Agreement for Nominal Scales. Educational and Psychological Measurement 20, 1 (1960), 37-46. https://doi.org/10.1177/001316446002000104 arXiv:https://doi.org/10.1177/001316446002000104

[8] Gilles Degottex, John Kane, Thomas Drugman, Tuomo Raitio, and Stefan Scherer. 2014. COVAREP - A collaborative voice analysis repository for speech technologies. IEEE, Florence, Italy, 960-964. https://github.com/covarep/covarep

[9] Abhinav Dhall, Roland Goecke, Simon Lucey, and Tom Gedeon. 2012. Collecting Large, Richly Annotated Facial-Expression Databases from Movies. IEEE MultiMedia 19, 3 (July 2012), 34-41. https://doi.org/10. 1109/MMUL.2012.26

[10] Eran Eidinger, Roee Enbar, and Tal Hassner. 2014. Age and Gender Estimation of Unfiltered Faces. Trans. Info. For. Sec. 9, 12 (Dec. 2014), 2170-2179. https://doi.org/10.1109/TIFS.2014.2359646

[11] Paul Ekman. 1992. An argument for basic emotions. Cognition and Emotion (1992), 169-200.

[12] P. Ekman. 1994. Strong evidence for universals in facial expressions: a reply to russell's mistaken critique. (1994).

[13] R H Finn. 1970. A Note on Estimating the Reliability of Categorical Data. Educational and Psychological Measurement 30 (1970), 71-76. https://doi.org/10/d8h9cp

[14] Mara Folster, Ursula Hess, and Katja Werheid. 2014. Facial age affects emotional decoding. Frontiers in psychology 5 (02 2014), 30. https: //doi.org/10.3389/fpsyg.2014.00030 
[15] Nir Friedman, Dan Geiger, and Moises Goldszmidt. 1997. Bayesian Network Classifiers. Mach. Learn. 29, 2-3 (Nov. 1997), 131-163. https: //doi.org/10.1023/A:1007465528199

[16] Markos Georgopoulos, Yannis Panagakis, and Maja Pantic. 2018. Modelling of Facial Aging and Kinship: A Survey. CoRR abs/1802.04636 (2018). arXiv:1802.04636 http://arxiv.org/abs/1802.04636

[17] R. Gockley, R. Simmons, and J. Forlizzi. 2006. Modeling Affect in Socially Interactive Robots. In ROMAN 2006 - The 15th IEEE International Symposium on Robot and Human Interactive Communication. 558-563. https://doi.org/10.1109/ROMAN.2006.314448

[18] Cindy Harmon-Jones, Brock Bastian, and Eddie Harmon-Jones. 2016. The Discrete Emotions Questionnaire: A New Tool for Measuring State Self-Reported Emotions. PLOS ONE 11, 8 (08 2016), 1-25. https: //doi.org/10.1371/journal.pone.0159915

[19] Larry V. Hedges. 1981. Distribution Theory for Glass's Estimator of Effect size and Related Estimators. Journal of Educational Statistics 6, 2 (1981), 107-128. https://doi.org/10.3102/10769986006002107 arXiv:https://doi.org/10.3102/10769986006002107

[20] Jean Kossaifi, Robert Walecki, Yannis Panagakis, Jie Shen, Maximilian Schmitt, Fabien Ringeval, Jing Han, Vedhas Pandit, Björn W. Schuller, Kam Star, Elnar Hajiyev, and Maja Pantic. 2019. SEWA DB: A Rich Database for Audio-Visual Emotion and Sentiment Research in the Wild. CoRR abs/1901.02839 (2019).

[21] Klaus Krippendorff. 2004. Content Analysis: An Introduction to Its Methodology (second edition). Sage Publications.

[22] Andreas Lanitis. 2008. Comparative Evaluation of Automatic Age Progression Methodologies. EURASIP J. Adv. Sig. Proc. 2008 (03 2008). https://doi.org/10.1155/2008/239480

[23] Changchun Liu, Pramila Agrawal, Nilanjan Sarkar, and Shuo Chen. 2009. Dynamic Difficulty Adjustment in Computer Games Through Real-Time Anxiety-Based Affective Feedback International Journal of Human-Computer Interaction 25, 6 (2009), 506-529. https://doi.org/10.1080/10447310902963944 arXiv:https://doi.org/10.1080/10447310902963944

[24] Carol Magai, Nathan S Consedine, Yulia S Krivoshekova, Elizabeth Kudadjie-Gyamfi, and Renee McPherson. 2006. Emotion experience and expression across the adult life span: Insights from a multimodal assessment study. Psychology and aging 21, 2 (2006), 303.

[25] Linda G. Martin. 1990. The status of South Asia's growing elderly population. Journal of Cross-Cultural Gerontology 5, 2 (01 Apr 1990), 93-117. https://doi.org/10.1007/BF00116568

[26] Stylianos Moschoglou, Athanasios Papaioannou, Christos Sagonas, Jiankang Deng, Irene Kotsia, and Stefanos Zafeiriou. 2017. Agedb: the first manually collected, in-the-wild age database. In Proceedings of the IEEE Conference on Computer Vision and Pattern Recognition Workshop, Vol. 2. 5.

[27] Gabriel Panis, Andreas Lanitis, Nicolas Tsapatsoulis, and Timothy F. Cootes. 2016. Overview of research on facial ageing using the FG-NET ageing database. IET Biometrics 5, 2 (2016), 37-46.

[28] Catherine Pelachaud. 2005. Multimodal Expressive Embodied Conversational Agents. In Proceedings of the 13th Annual ACM International Conference on Multimedia (MULTIMEDIA '05). ACM, New York, NY, USA, 683-689. https://doi.org/10.1145/1101149.1101301

[29] Thomas S. Polzin and Alexander Waibel. 2000. EMOTION-SENSITIVE HUMAN-COMPUTER INTERFACES. ITRW on Speech and Emotion (2000).

[30] Allen Rawls and Karl Ricanek. 2009. MORPH: Development and Optimization of a Longitudinal Age Progression Database. In COST 2101/2102 Conference (Lecture Notes in Computer Science), Vol. 5707. Springer, 17-24.
[31] K. Ricanek and T. Tesafaye. 2006. MORPH: a longitudinal image database of normal adult age-progression. In 7th International Conference on Automatic Face and Gesture Recognition (FGR06). 341-345. https://doi.org/10.1109/FGR.2006.78

[32] Fabien Ringeval, Andreas Sonderegger, Jurgen S. Sauer, and Denis Lalanne. 2013. Introducing the RECOLA multimodal corpus of remote collaborative and affective interactions.. In FG. IEEE Computer Society, 1-8. http://dblp.uni-trier.de/db/conf/fgr/fg2013.html\#RingevalSSL13

[33] Rasmus Rothe, Radu Timofte, and Luc Van Gool. 2016. Deep expectation of real and apparent age from a single image without facial landmarks. International fournal of Computer Vision (IFCV) (July 2016).

[34] N. Sato and Y. Obuchi. 2007. Emotion recognition using mel-frequency cepstral coefficients. (2007).

[35] Patrick Shrout and Joseph L. Fleiss. 1979. Intraclass correlations: Uses in assessing rater reliability. Psychological Bulletin 86, 2 (3 1979), 420428. https://doi.org/10.1037/0033-2909.86.2.420

[36] Maxim Sidorov and Wolfgang Minker. 2014. Emotion Recognition and Depression Diagnosis by Acoustic and Visual Features: A Multimodal Approach. In Proceedings of the 4th International Workshop on Audio/Visual Emotion Challenge (AVEC '14). ACM, New York, NY, USA, 81-86. https://doi.org/10.1145/2661806.2661816

[37] Olga Sourina, Yisi Liu, and Minh Khoa Nguyen. 2012. Real-time EEGbased emotion recognition for music therapy. fournal on Multimodal User Interfaces 5, 1 (01 Mar 2012), 27-35. https://doi.org/10.1007/ s12193-011-0080-6

[38] J. A. K. Suykens and J. Vandewalle. 1999. Least Squares Support Vector Machine Classifiers. Neural Process. Lett. 9, 3 (June 1999), 293-300. https://doi.org/10.1023/A:1018628609742

[39] D. Tacconi, O. Mayora, P. Lukowicz, B. Arnrich, C. Setz, G. Troster, and C. Haring. [n. d.]. Activity and emotion recognition to support early diagnosis of psychiatric diseases. In 2008 Second International Conference on Pervasive Computing Technologies for Healthcare.

[40] Michel Valstar, Björn Schuller, Kirsty Smith, Florian Eyben, Bihan Jiang, Sanjay Bilakhia, Sebastian Schnieder, Roddy Cowie, and Maja Pantic. 2013. AVEC 2013: The Continuous Audio/Visual Emotion and Depression Recognition Challenge. In Proceedings of the 3rd ACM International Workshop on Audio/Visual Emotion Challenge (AVEC '13). ACM, New York, NY, USA, 3-10. https://doi.org/10.1145/2512530. 2512533

[41] H. Wang, A. Meghawat, L. Morency, and E. P. Xing. 2017. Selectadditive learning: Improving generalization in multimodal sentiment analysis. In 2017 IEEE International Conference on Multimedia and Expo (ICME). 949-954. https://doi.org/10.1109/ICME.2017.8019301

[42] KX Wang, QL Zhang, and SY Liao. [n. d.]. A database of elderly emotional speech.

[43] Kunxia Wang, ZongBao Zhu, Shidong Wang, Xiao Sun, and Lian Li. 2016. A database for emotional interactions of the elderly. 1-6. https: //doi.org/10.1109/ICIS.2016.7550902 